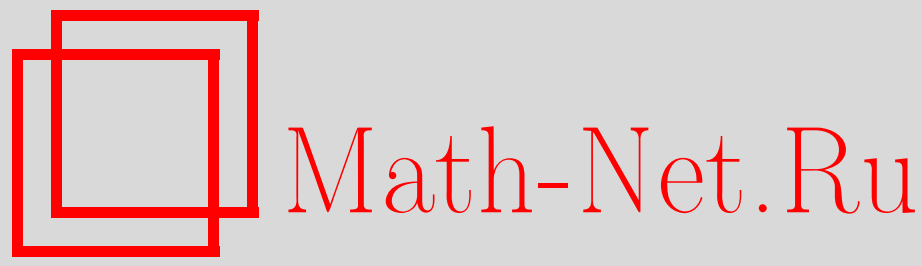

И. В. Волович, Уравнения Боголюбова и функциональная механика, TMФ, 2010, том 164, номер 3, 354-362

DOI: https://doi.org/10.4213/tmf6544

Использование Общероссийского математического портала Math-Net.Ru подразумевает, что вы прочитали и согласны с пользовательским соглашением http: //www.mathnet.ru/rus/agreement

Параметры загрузки :

IP: 54.224 .187 .69

26 апреля 2023 г., 15:39:17

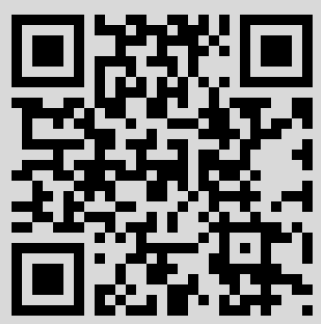




\title{
УРАВНЕНИЯ БОГОЛЮБОВА И ФУНКЦИОНАЛЬНАЯ МЕХАНИКА
}

\begin{abstract}
Для решения проблемы необратимости, т. е. проблемы согласования обратимости во времени уравнений микроскопической динамики с необратимостью динамики макросистем, недавно была предложена функциональная классическая механика, основанная на вероятностном подходе, когда частица описывается не траекторией в фазовом пространстве, а вероятностным распределением. В настоящей работе в рамках функциональной механики для конечного числа частиц дается вывод уравнений типа Боголюбова-Больцмана. Показано, что в функциональной механике замкнутое уравнение для одночастичной функции распределения может быть строго получено без дополнительных предположений, необходимых в методе Боголюбова. Рассматривается возможность описания изолированных частиц при помощи диффузионных процессов и уравнения Фоккера-Планка-Колмогорова.
\end{abstract}

Ключевые слова: уравнение Больцмана, уравнение Боголюбова, кинетическая теория.

\section{1. ВВЕДЕНИЕ}

В замечательной работе Боголюбова [1] был предложен метод получения кинетических уравнений из уравнений механики Ньютона. Основные этапы метода следующие: выписывается уравнение Лиувилля для функции распределения для частиц в конечном объеме, выводится цепочка уравнений для функций распределения, производится предельный переход к бесконечному объему, постулируется условие ослабления начальных корреляций между частицами в отдаленном прошлом, предполагается наличие кинетической и гидродинамической стадий эволюции, вводится гипотеза о зависимости всех многочастичных функций рапределения от времени только через одночастичную функцию распределения, используется формальное разложение по степеням плотности. Отметим, что эта сложная и красивая теория приводит к расходимостям в высших порядках разложения по степеням плотности.

В методе Боголюбова необратимость по времени вводится при помощи условия ослабления начальных корреляций, т. е. через граничные условия. Напомним, что

${ }^{*}$ Математический институт им. В. А. Стеклова РАН, Москва, Россия. E-mail: volovich@mi.ras.ru 
проблема необратимости формулируется следующим образом: почему в макроскопической системе происходит необратимый процесс, тогда как динамические законы движения отдельных молекул обратимы по времени? Требуется установить соответствие между симметрией относительно обращения времени микроскопических уравнений движения Ньютона классической механики и необратимым поведением макроскопических систем, описываемых уравнениями диффузии, Навье-Стокса, Больцмана и другими, когда имеет место термодинамический закон возрастания энтропии. Аналогичная проблема необратимости имеет место и в квантовой механике.

Проблема необратимости изучалась во многих работах (см., например, [2]-[12]), однако эта проблема заслуживает дальнейшего изучения. В частности, в работах Пуанкаре [3], Ландау и Лифшица [5], Гинзбурга [10], Фейнмана [12] подчеркивается, что проблема необратимости остается открытой.

При микроскопическом механическом описании системы предполагается, что состояние системы в некоторый момент времени задается точкой в фазовом пространстве с инвариантной мерой; эволюция системы с течением времени описывается траекторией в фазовом пространстве (см. [13], [14]). Обычно предполагается, что микроскопические математические законы движения нам известны (уравнения Ньютона или Шредингера), и ставится задача вывести из них приближенные макроскопические уравнения (см., например, [5], [6]), при этом возникает проблема необратимости [5].

Отметим, однако, что широко используемое понятие микроскопического состояния системы в некоторый момент времени как точки в фазовом пространстве, а также понятия траектории и микроскопических уравнений движения не имеют непосредственного физического смысла, поскольку произвольные вещественные числа ненаблюдаемы. Наблюдаемыми являются только физические величины, представляемые с определенной точностью рациональными числами (см. обсуждение в [15]-[18]).

В работах [19]-[21] предложена попытка решения проблемы необратимости при помощи модификации ньютоновского подхода к классической механике - предлагается вероятностный подход к механике, когда частица описывается не траекторией в фазовом пространстве, а вероятностным распределением.

Парадоксы Лошмидта и Пуанкаре-Цермело в предложенном неньютоновском подходе не возникают, поскольку необратимость в определенном смысле имеет место уже на микроскопическом уровне. Таким образом, снимается противоречие между обратимостью микроскопической и необратимостью макроскопической динамики, поскольку и микроскопическая, и макроскопическая динамики в предлагаемом подходе необратимы (см. обсуждение этих вопросов в работах [20], [22], [23], а также в разделе 4 настоящей статьи).

В функциональном подходе [19]-[21] физический смысл придается не отдельной траектории, а только "пучку" траекторий либо функции распределения на фазовом пространстве. Фундаментальным уравнением микроскопической динамики теперь является не уравнение Ньютона, а уравнение Лиувилля для функции распределения, решения которого обладают свойством делокализации, что соответствует 
необратимости. Показано, что уравнение Ньютона в таком подходе возникает как приближенное уравнение, описывающее динамику средних значений координат и импульсов для не слишком больших промежутков времени, и вычислены поправки к уравнению Ньютона. В разделе 4 мы рассматриваем также более общий подход.

В работе Боголюбова и Крылова [24] подчеркивается, что в механике имеется неопределенность не только в начальных данных, но и сам вид уравнений движения известен только приближенно. В этой и последующих работах была развита вероятностная эргодическая теория. В дальнейшем аналогичные идеи обсуждались в работах [25], [7]. Купман [26] предложил использовать методы гильбертова пространства в классической механике, такой подход широко применяется в эргодической теории. Однако, по-видимому, не ставился вопрос об отказе от уравнений Ньютона и траекторного описания на фундаментальном уровне (даже для одной изолированной частицы в пустоте), не рассматривалось вычисление поправок к средним ньютоновским траекториям и, таким образом, не предлагалось решать проблему необратимости, как это предлагается в функциональном подходе [19]-[21].

В работах Козлова [8], [9] был разработан метод слабого предела в неравновесной статистической механике, позволивший, в частности, доказать, что для ряда моделей система в смысле слабой сходимости необратимо стремится к одному и тому же состоянию статистического равновесия как в прошлом, так и в будущем.

В разделе 2 приводятся основные понятия функциональной формулировки классической механики, определены состояния и наблюдаемые в этом подходе. В разделе 3 дан вывод уравнения типа Боголюбова для двух частиц в конечном объеме в функциональной механике. В разделе 4 рассмотрен более общий подход, когда динамика одной изолированной частицы описывается уравнением типа Фоккера-Планка-Колмогорова. Как хорошо известно, обычно это уравнение применяется для описания движения частицы в среде.

\section{2. ФУНКЦИОНАЛЬНАЯ ФОРМУЛИРОВКА КЛАССИЧЕСКОЙ МЕХАНИКИ}

В классической ньютоновской механике движение точечного тела описывается заданием траектории в фазовом пространстве, т. е. значений координаты и импульса как функций времени, которые находятся как решения уравнений Ньютона или Гамильтона. Заметим, однако, что такая математическая модель является идеализированной моделью, далеко отстоящей от реальности. Физическое тело всегда имеет пространственные размеры, поэтому математическая точка дает только приближенное описание. Далее, математическое понятие траектории также не имеет непосредственного физического смысла, поскольку использует представление о вещественных числах, т. е. о бесконечных десятичных дробях, тогда как наблюдать возможно в лучшем случае только рациональные числа, да и то с некоторой погрешностью. Поэтому в “функциональном" подходе [19]-[21] к классической механике состояние системы материальных точек описывается не точкой в фазовом пространстве, а вероятностным распределением. 
Рассмотрим движение одной классической частицы в трехмерном пространстве. Пусть $(q, p)$ - координаты в пространстве $\mathbb{R}^{3} \times \mathbb{R}^{3}$ (фазовом пространстве), $t \in \mathbb{R}-$ время. Состояние классической частицы в момент времени $t$ будем описывать функцией $\rho=\rho(q, p, t)$, которая представляет собой плотность вероятности того, что частица в момент времени $t$ имеет координату $q$ и импульс $p$.

Описание механической системы при помощи вероятностной функции распределения $\rho=\rho(q, p, t)$ не обязательно означает, что мы имеем дело с ансамблем множества одинаково приготовленных частиц. Мы можем использовать описание при помощи функции $\rho=\rho(q, p, t)$ и для индивидуального тела. Фактически неявно так всегда и делается, при этом функция $\rho=\rho(q, p, t)$ учитывает неизбежные погрешности в определении координаты и импульса тела [22].

Конкретный вид функции $\rho$ зависит от способа приготовления состояния классической частицы в начальный момент времени и от вида потенциального поля. Если функция $\rho(q, p, t)$ имеет острые пики при $q=q_{0}$ и $p=p_{0}$, мы говорим, что частица имеет приближенные значения координаты и импульса $q_{0}$ и $p_{0}$.

Подчеркнем, что абсолютно точное определение координаты и импульса невозможно не только в квантовой механике, где имеется соотношение неопределенностей Гейзенберга, но и в классической механике. Всегда имеется некоторая погрешность в задании координат и импульсов. Можно утверждать, что имеются классические соотношения неопределенностей - погрешности в определении значений координаты и импульса всегда строго больше нуля:

$$
\Delta q>0, \quad \Delta p>0 .
$$

Понятие вещественного числа, заданного бесконечной дробью, является математической идеализацией, такие числа невозможно измерить в эксперименте. Поэтому в предлагаемом функциональном подходе к классической механике понятие точной траектории частицы отсутствует, исходным понятием является функция распределения $\rho=\rho(q, p, t)$, причем $\delta$-функция в качестве функции распределения недопустима.

Предполагается, что непрерывно дифференцируемая и интегрируемая функция $\rho=\rho(q, p, t)$ удовлетворяет следующим условиям:

$$
\rho \geqslant 0, \quad \int_{\mathbb{R}^{6}} \rho(q, p, t) d q d p=1, \quad t \in \mathbb{R} .
$$

Формулировка классической механики на языке состояний и наблюдаемых описана в [27]. Функциональный подход к классической механике отличается в следующих отношениях. Поскольку понятие точной траектории частицы в функциональном подходе отсутствует, то функция $\rho=\rho(q, p, t)$ не может быть обобщенной функцией, а является обыкновенной функцией класса $L^{1}\left(\mathbb{R}^{6}\right)$ или даже непрерывно дифференцируемой и интегрируемой функцией. Кроме того, движение частицы в функциональном подходе не описывается непосредственно уравнением Ньютона (Гамильтона). Уравнение Ньютона в функциональном подходе выступает как приближенное уравнение для средних координат частиц, причем в случае нелинейной динамики к нему имеются поправки. 
Наблюдаемыми для системы частиц или даже для одной частицы в функциональном подходе к классической механике являются не индивидуальные траектории, а средние с функцией распределения $\rho$. Если $f=f(q, p)$ - функция на фазовом пространстве, то ее среднее значение $\bar{f}$ в момент времени $t$ задается интегралом

$$
\bar{f}(t)=\int f(q, p) \rho(q, p, t) d q d p .
$$

В некотором смысле мы имеем дело со случайным процессом $\xi(t)$ со значениями в фазовом пространстве.

\section{3. ВЫВОД УРАВНЕНИЯ ТИПА БОГОЛЮБОВА В ФУНКЦИОНАЛЬНОЙ МЕХАНИКЕ}

Рассмотрим систему из $N$ одинаковых частиц, заключенных в некоторой ограниченной области $G$ в пространстве $\mathbb{R}^{3}$ с гладкой границей объема $V$. Фазовое пространство одной молекулы определяется как пространство точек $x=(q, p) \in \Omega_{V}=$ $G \times \mathbb{R}^{3}$. Уравнение Лиувилля для функции распределения $\rho=\rho\left(x_{1}, x_{2}, \ldots, x_{N}, t\right)$ имеет вид

$$
\frac{\partial \rho}{\partial t}=\sum_{i, \alpha}\left(\frac{\partial U(q)}{\partial q_{i}^{\alpha}} \frac{\partial \rho}{\partial p_{i}^{\alpha}}-\frac{p_{i}^{\alpha}}{m} \frac{\partial \rho}{\partial q_{i}^{\alpha}}\right),
$$

где суммирование идет по $i=1, \ldots, N, \alpha=1,2,3$. Функция $\rho=\rho\left(x_{1}, x_{2}, \ldots, x_{N}, t\right)$ предполагается непрерывно дифференцируемой по всем аргументам, интегрируемой по $x_{i} \in \Omega_{V}$, удовлетворяющей условиям

$$
\int_{\Omega_{V}} \ldots \int_{\Omega_{V}} \rho\left(x_{1}, x_{2}, \ldots, x_{N}, t\right) d x_{1} \ldots d x_{N}=1, \quad t \in \mathbb{R}, \quad \rho \geqslant 0 .
$$

Потенциальная энергия имеет вид суммы парных потенциалов:

$$
U(q)=\sum_{1 \leqslant i<j \leqslant N} \Phi\left(\left|q_{i}-q_{j}\right|\right),
$$

где $\Phi\left(\left|q_{i}-q_{j}\right|\right)$ - непрерывно дифференцируемая, интегрируемая функция. К потенциалу (5) иногда добавляется еще потенциальная функция $U_{G}\left(q_{i}\right)$, действующая на каждую частицу, постоянная внутри области $G$ и резко возрастающая к бесконечности при приближении к границе области.

Уравнения характеристик для уравнения в частных производных первого порядка (3) суть уравнения Гамильтона

$$
\dot{q}_{i}^{\alpha}=\frac{\partial H}{\partial p_{i}^{\alpha}}, \quad \dot{p}_{i}^{\alpha}=-\frac{\partial H}{\partial q_{i}^{\alpha}},
$$

где гамильтониан

$$
H=\sum_{i, \alpha} \frac{\left(p_{i}^{\alpha}\right)^{2}}{2 m}+U(q)
$$

Подчеркнем еще раз, что уравнения Гамильтона (6) в рассматриваемом функциональном подходе к механике не описывают непосредственно движение системы 
частиц, а являются лишь уравнениями характеристик для имеющего физический смысл уравнения Лиувилля (3). Средние значения координаты и импульса вычисляются по формуле (2). Уравнение Лиувилля (3) можно записать в виде

$$
\frac{\partial \rho}{\partial t}=\{H, \rho\}
$$

где скобка Пуассона

$$
\{H, \rho\}=\sum_{i, \alpha}\left(\frac{\partial H}{\partial q_{i}^{\alpha}} \frac{\partial \rho}{\partial p_{i}^{\alpha}}-\frac{\partial H}{\partial p_{i}^{\alpha}} \frac{\partial \rho}{\partial q_{i}^{\alpha}}\right) .
$$

Рассмотрим систему из двух частиц в функциональной механике. Уравнение Лиувилля для функции распределения $\rho=\rho\left(x_{1}, x_{2}, t\right)$ имеет вид

$$
\frac{\partial \rho}{\partial t}=\frac{\partial \Phi\left(\left|q_{1}-q_{2}\right|\right)}{\partial q_{1}} \frac{\partial \rho}{\partial p_{1}}-\frac{p_{1}}{m} \frac{\partial \rho}{\partial q_{1}}+\frac{\partial \Phi\left(\left|q_{1}-q_{2}\right|\right)}{\partial q_{2}} \frac{\partial \rho}{\partial p_{2}}-\frac{p_{2}}{m} \frac{\partial \rho}{\partial q_{2}} .
$$

Здесь используются векторные обозначения, в частности

$$
\frac{p_{1}}{m} \frac{\partial \rho}{\partial q_{1}}=\sum_{\alpha} \frac{p_{1}^{\alpha}}{m} \frac{\partial \rho}{\partial q_{1}^{\alpha}} .
$$

Предполагается, что функция $\rho$ симметрична относительно перестановки аргументов: $\rho\left(x_{1}, x_{2}, t\right)=\rho\left(x_{2}, x_{1}, t\right)$. Следуя Боголюбову, введем функции

$$
f_{1}\left(x_{1}, t\right)=V \int_{\Omega_{V}} \rho\left(x_{1}, x_{2}, t\right) d x_{2}, \quad f_{2}\left(x_{1}, x_{2}, t\right)=V^{2} \rho\left(x_{1}, x_{2}, t\right),
$$

где $x_{1}=\left(q_{1}, p_{1}\right), x_{2}=\left(q_{2}, p_{2}\right)$. Умножим соотношение $(10)$ на $V$ и проинтегрируем по $d q_{2} d p_{2}$. Предполагая, что функция распределения исчезает на границе области $G$ (для биллиардов появятся дополнительные слагаемые), получим

$$
\left(\frac{\partial}{\partial t}+\frac{p_{1}}{m} \frac{\partial}{\partial q_{1}}\right) f_{1}\left(x_{1}, t\right)=\frac{1}{V} \int_{\Omega_{V}} \frac{\partial \Phi\left(\left|q_{1}-q_{2}\right|\right)}{\partial q_{1}} \frac{\partial f_{2}\left(x_{1}, x_{2}, t\right)}{\partial p_{1}} d q_{2} d p_{2} .
$$

Предположим, что в некоторый момент времени $t_{0}$ двухчастичная функция распределения имеет вид произведения одночастичных функций распределения:

$$
f_{2}\left(x_{1}, x_{2}, t_{0}\right)=f_{1}\left(x_{1}, t_{0}\right) f_{1}\left(x_{2}, t_{0}\right) .
$$

Пусть $\varphi_{t}\left(x_{1}, x_{2}\right)$ обозначает перенос точки $\left(x_{1}, x_{2}\right)$ потоком в фазовом пространстве вдоль решений уравнений характеристик. Тогда в силу (12) решение уравнения Лиувилля примет вид

$$
f_{2}\left(x_{1}, x_{2}, t\right)=f_{1}\left(\varphi_{t_{0}-t}^{(1)}\left(x_{1}, x_{2}\right), t_{0}\right) f_{1}\left(\varphi_{t_{0}-t}^{(2)}\left(x_{1}, x_{2}\right), t_{0}\right),
$$

где индекс (1) или (2) над $\varphi$ означает проекцию на первую или вторую координату. Подставив выражение (13) в (11), получим

$$
\begin{aligned}
\left(\frac{\partial}{\partial t}\right. & \left.+\frac{p_{1}}{m} \frac{\partial}{\partial q_{1}}\right) f_{1}\left(x_{1}, t\right)=\frac{1}{V} \int_{\Omega_{V}} \frac{\partial \Phi\left(\left|q_{1}-q_{2}\right|\right)}{\partial q_{1}} \times \\
& \times \frac{\partial}{\partial p_{1}}\left[f_{1}\left(\varphi_{t_{0}-t}^{(1)}\left(x_{1}, x_{2}\right), t_{0}\right) f_{1}\left(\varphi_{t_{0}-t}^{(2)}\left(x_{1}, x_{2}\right), t_{0}\right)\right] d q_{2} d p_{2}
\end{aligned}
$$


Уравнение (14) является нелинейным интегродифференциальным уравнением для функции $f_{1}$, мы будем называть его уравнением типа Боголюбова. Чтобы получить из него уравнение Больцмана, нужно сделать дополнительные преобразования, аналогичные методу Боголюбова, описанному в начале раздела 1 , математически обосновать которые не представляется возможным. Существенным, в частности, является переход к пределу бесконечного объема. В функциональной механике в настоящей работе мы рассматриваем систему двух частиц в конечном объеме, поэтому для исследования динамики существенным является именно уравнение (14). Никаких дополнительных предположений, кроме условия на начальные данные (12), при выводе уравнения не делалось. Итак, доказано следующее

ПреДЛОЖЕНИЕ. Пусть дана система из двух частии в области G в функииональной классической механике. Тогда из уравнения Лиувилля для двухчастичной функции распределения при условии на начальные данные (12) следует уравнение типа Боголюбова (14) для одночастичной функции распределения $f_{1}$.

Рассмотрение асимптотических свойств решений уравнения (14) и исследование связи этого уравнения с уравнением Больцмана планируется выполнить в отдельной работе.

\section{4. НЕОБРАТИМОСТЬ И ДИФФУЗИОННЫЕ ПРОЦЕССЫ}

Известные аргументы об обратимости движения в классической механике относятся к индивидуальной траектории точечной частицы и не применимы непосредственно к функциональной механике, поскольку состояние частицы описывается здесь функцией распределения. Тем не менее, поскольку функция распределения удовлетворяет уравнению Лиувилля, которое симметрично относительно обращения времени, обратимость может иметь место в некотором приближении, как это было показано Трушечкиным [23].

В функциональной механике функция распределения учитывает неизбежные неопределенности в определении координат и импульсов частиц. Отметим также, что не только наблюдаемая частица, но и приборы наблюдателя испытывают флуктуации разнообразной природы - тепловые, вакуумные, полевые и т.д. Рассмотрим возможность более общего описания движения частиц, чем при помощи уравнения Лиувилля. Естественное обобщение уравнения Лиувилля - это уравнение Фоккера-Планка-Колмогорова, которое в изотропном случае имеет вид

$$
\frac{\partial f}{\partial t}-\{H, f\}=\sigma \sum_{i} \frac{\partial^{2} f}{\partial p_{i}^{2}}+\gamma \sum_{i} \frac{\partial\left(p_{i} f\right)}{\partial p_{i}}
$$

где $f=f(q, p, t)$, а $\gamma$ и $\sigma$ - константы трения и диффузии соответственно. Если гамильтониан имеет вид $H=\sum_{i} p_{i}^{2} /(2 m)+U(q)$, то стационарным решением этого уравнения является распределение Гиббса: $\rho_{0}(q, p)=$ const $\cdot e^{-m \gamma H / \sigma}$. Для свободной частицы имеем

$$
\frac{\partial f}{\partial t}+\frac{p}{m} \frac{\partial f}{\partial q}=\sigma \frac{\partial^{2} f}{\partial p^{2}}+\gamma \frac{\partial(p f)}{\partial p} .
$$


Обычно уравнение Фоккера-Планка-Колмогорова используется для учета влияния на частицу окружающей среды [28]-[30]. Однако даже если рассматривается одна частица в вакууме, флуктуации координаты и импульса неизбежны вследствие макроскопичности приборов наблюдателя. Такой подход, по-видимому, находится в согласии с идеей Боголюбова и Крылова [24], что вид уравнений известен нам только приближенно.

Уравнение (16) несимметрично относительно обращения времени, временная эволюция решений этого уравнения приводит к распределению Максвелла.

Уравнение Фоккера-Планка-Колмогорова определяет диффузионный процесс [30]. Было бы интересно распространить подход функциональной механики на случай более общих диффузионных и марковских процессов. Вопрос об обратимости диффузионных процессов рассматривался Колмогоровым [4].

\section{5. ЗАКЛЮЧЕНИЕ}

В настоящей работе в рамках функциональной механики, когда за основу математического описания механической системы берутся не уравнения Ньютона, а уравнение для функции распределения или для функции перехода, дан вывод уравнения типа Боголюбова. Показано, что в функциональной механике замкнутое уравнение для одночастичной функции распределения может быть строго получено без дополнительных предположений, необходимых в методе Боголюбова. Рассмотрена возможность описания изолированных частиц при помощи диффузионных процессов и уравнения Фоккера-Планка-Колмогорова. В функциональном подходе имеются поправки к средним траекториям частиц, определяемым из уравнений Ньютона. Представляет интерес изучение возможности объяснения аномального поведения траекторий, обнаруженного в проектах Pioneer и Rosetta [31], с использованием этих поправок к траекториям Ньютона.

Благодарности. Автор выражает искреннюю благодарность Д.В. Аносову, В. С. Владимирову, А.И. Кириллову, В.В.Козлову, Г. Аденье, Е. В. Писковскому, А. Д. Суханову, А. С. Трушечкину, А. Н. Ширяеву и участникам семинара по проблеме необратимости НОЩ МИАН за плодотворные обсуждения. Работа частично поддержана грантами НШ-7675.2010.1, РФФИ № 08-01-00727-а, 09-01-12161-офи-м и программой ОМН РАН.

\section{Список литературы}

[1] Н.Н. Боголюбов, Проблемы динамической теории в статистической физике, Гостехиздат, М., Л., 1946.

[2] Л. Больцман, Лекиии по теории газов, Гостехиздат, М., 1953.

[3] А. Пуанкаре, "Механицизм и опыт": Л. Больцман, Избранные труды, Наука, М., 1984, 434-437.

[4] A. Kolmogoroff, Math. Ann., 113:1 (1937), 766-772.

[5] Л. Д. Ландау, Е. М. Лифшиц, Теоретическая физика. т. 5: Статистическая физика. Ч. 1. Теория равновесных систем, Наука, М., 1964.

[6] Д. Н. Зубарев, Неравновесная статистическая термодинамика, Физматлит, М., 1971.

[7] I. Prigogine, Les Lois du Chaos, Flammarion, Paris, 1993. 
[8] В. В. Козлов, Тепловое равновесие по Гиббсу и Пуанкаре, Современная математика, РХД, М., Ижевск, 2002.

[9] В. В. Козлов, Ансамбли Гиббса и неравновесная статистическая механика, НИЦ "Регулярная и хаотическая динамика", М.-Ижевск, 2008.

[10] В.Л. Гинзбург, "Какие проблемы физики и астрофизики представляются особенно важными и интересными в начале XXI века?", О науке, о себе и о других, Физматлит, M., 2003, 11-74.

[11] А. Д. Суханов, ЭЧАЯ, 36:6 (2005), 1281-1342.

[12] Р. Фейнман, Характер физических законов, Наука, М., 1987.

[13] В. И. Арнольд, Математические методы классической механики, Наука, М., 1989.

[14] Б. А. Дубровин, С.П. Новиков, А. Т. Фоменко, Современная геометрия. Методъ и приложения. т. 2: Геометрия и топология многообразий, Эдиториал УРСС, М., 1986.

[15] I. V. Volovich, Number theory as the ultimate physical theory, Preprint No. TH 4781/87, CERN, Geneva, 1987; p-Adic Numbers Ultrametric Anal. Appl., 2:1 (2010), 77-87.

[16] И. В. Волович, ТМФ, 71:3 (1987), 337-340.

[17] В. С. Владимиров, И. В. Волович, Е. И. Зеленов, p-Адический анализ и математическал физика, Наука, М., 1994.

[18] B. Dragovich, A. Yu. Khrennikov, S. V. Kozyrev, I. V. Volovich, P-Adic Numbers, Ultrametric Anal. Appl., 1:1 (2009), 1-17, arXiv: 0904.4205.

[19] И. В. Волович, Вестн. СамГУ, 8/1:67 (2008), 35-55.

[20] I. V. Volovich, Time irreversibility problem and functional formulation of classical mechanics, arXiv: 0907.2445.

[21] I. V. Volovich, "Randomness in classical mechanics and quantum mechanics", Found. Phys., 2010 (in press).

[22] A.S. Trushechkin, I. V. Volovich, P-Adic Numbers Ultrametric Anal. Appl., 1:4 (2009), 361-367, arXiv: 0910.1502.

[23] А. С. Трушечкин, ТМФ, 164:3 (2010), 435-440.

[24] Н. Н. Боголюбов, Н. М. Крылов, "Наслідки діi статистичноі зміни параметрів на рух динамічних консервативних систем протягом досить тривалих перідів часу”, Зб. праць з нелінійноі механіки, Вид-во АН УРСР, Киів, 1937, 119-135; Н. Н. Боголюбов, Математика и нелинейная механика, Собр. научных трудов. т. II: Нелинейная механика, ред. Ю. А. Митропольский, А. Д. Суханов, Наука, М., 2005, 730-736.

[25] M. Born, Z. f. Phys., 153:3 (1958), 372-388.

[26] B. O. Koopman, Proc. Natl. Acad. Sci. USA, 17:5 (1931), 315-318.

[27] Л. Д. Фаддеев, О.А.Якубовский, Лекции по квантовой механике для студентов математиков, ЛГУ, Л., 1980.

[28] В. С. Владимиров, Уравнения математической физики, Наука, М., 1988.

[29] L. Accardi, Y. G. Lu, I. V. Volovich, Quantum Theory and its Stochastic Limit, Texts Monogr. Phys., Springer, Berlin, 2002.

[30] Ю. В. Прохоров, Ю. А. Розанов, Теория вероятностей, Наука, М., 1987.

[31] J. D. Anderson, J. K. Campbell, J. E. Ekelund, J. Ellis, J. F. Jordan, Phys. Rev. Lett., 100:9 (2008), 091102, 4 pp. 